\title{
A Case Report of Bulky Venous Malformation in the Parapharyngeal Space
}

\author{
Yuki Sato $^{1}$ Takao Hamamoto $^{2}$ Takashi Ishino $^{2}$ Tsutomu Ueda ${ }^{2}$ Masay Takumida ${ }^{2}$ Sachio Takeno $^{2}$ \\ ${ }^{1}$ Department of Otorhinolaryngology, Hiroshima Prefectural \\ Hospital, Hiroshima City, Hiroshima, Japan \\ 2 Department of Otorhinolaryngology, Head and Neck Surgery, \\ Hiroshima University Hospital, Hiroshima City, Hiroshima, Japan

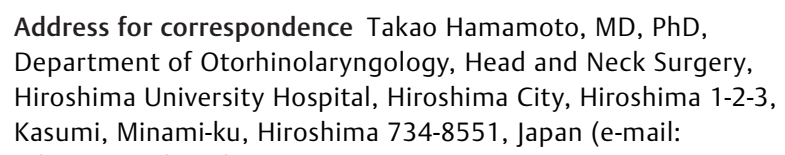

Address for correspondence Takao Hamamoto, MD, PhD, Department of Otorhinolaryngology, Head and Neck Surgery, Hiroshima University Hospital, Hiroshima City, Hiroshima 1-2-3, Kasumi, Minami-ku, Hiroshima 734-8551, Japan (e-mail: \\ takao0320@hiroshima-u.ac.jp).
}

Int J Pract Otolaryngol 2020;3:e10-e15.

\author{
Abstract \\ Keywords \\ - venous malformation \\ - head and neck \\ - parapharyngeal space
}

Venous malformations commonly occur in the head and neck regions, in the mucous membrane of the nasal and oral cavities. Venous malformations are found in the extremities in $40 \%$ of cases, in the trunk in $20 \%$ of cases, and in the cervicofacial area in $40 \%$ of cases. However, they are rarely encountered in the parapharyngeal space. We report our experience of surgical treatment of a patient with this rare tumor. The patient was a 21-year-old man who presented with a left mandibular swelling and was admitted to a nearby hospital. He was referred to our hospital for examination and treatment. Contrast-enhanced computed tomography (CT) revealed a bulky tumor with some calcification in the parapharyngeal space; on contrast-enhanced $\mathrm{CT}$, the tumor showed some enhanced effects in the arterial and venous phases. Additional enhancement magnetic resonance imaging (MRI) showed the lesion as low signal intensity on T1-weighted images and as high signal intensity on T2-weighted images, and contrast-enhanced MRI showed some enhanced effects of the lesion. Based on these findings, we suspected venous malformation and performed surgical excision. Histopathology confirmed the venous malformation, and the lesion was diagnosed as a cavernous angioma. Vascular malformations in the head and neck regions should be precisely diagnosed so that appropriate multimodality treatment can be undertaken.

\section{Introduction}

Historically, vascular anomalies have been labeled with descriptive terms, according to the food they resembled (port wine, strawberry, cherry, salmon patch). This imprecise terminology has caused diagnostic confusion, blocked communication, and even caused incorrect treatment, as it does not differentiate between various vascular anomalies. ${ }^{1}$

In 1982, a classification was established by the International Society for the Study of Vascular Anomalies (ISSVA). ${ }^{2}$ This classification divides vascular anomalies into vascular tumors and vascular malformations. Hemangioma is the most common vascular tumor, which usually appears postnatally, exhibiting rapid growth due to cellular proliferation, followed by

received

May 15, 2019

accepted

February 28, 2020
DOI https://doi.org/

10.1055/s-0040-1713649. ISSN 2569-1783. inevitable involution. In contrast, vascular malformations are present at birth and grow proportionally with the patient. They are subdivided depending on the affected vessel type into capillary, venous, lymphatic, and arterial malformations. ${ }^{3,4}$

Although vascular malformations are one of the most common soft-tissue malformations of the head and neck, they are relatively rare in the parapharyngeal space. ${ }^{1}$ Herein, we describe a case report of barky venous malformation in the parapharyngeal space with a review of literatures.

\section{Case Report}

A 21-year-old man who had no particular self or family medical history experienced pain in the left mandibular space

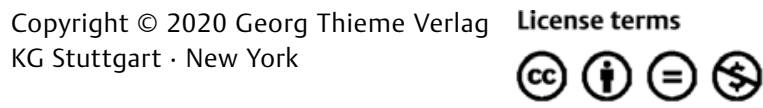


and referred to a nearby hospital. Physical examination revealed an elastic, hard, unmovable tumor existing in left mandibular space without tenderness, which is an abnormal neurological finding. There was no obvious change of the tumor findings upon increasing venous pressure via Valsalva maneuver.

Magnetic resonance imaging (MRI) was performed and detected a huge tumor located in the left parapharyngeal space. For additional precise examination and treatment, the patient was referred to our hospital. Flexible laryngoscopy findings showed nasopharyngeal submucosal swelling beneath the tumor and no mucosal lesion. Blood chemistry study showed acceptable limits. Neck ultrasonography revealed a hypoechoic, hypervascular, and clear marginal tumor located in the submandibular space with partial calcifications. There were no remarkable inflow or outflow vessels. Contrast-enhanced computed tomography (CT) scan revealed a bulky tumor with some calcified lesion located in the parapharyngeal space. The tumor had some enhanced effects in both arterial- and venous-enhanced phases (-Fig. 1). Additional enhanced MRI showed low signal intensity on T1 and high signal intensity on T2 and some effects of enhancement (-Fig. 2). Although fine needle aspiration was performed, bloody drainage was obtained and it had no epithelial component. Based on these findings, we suspected the tumor to be a venous malformation. We informed details of the examination findings and suggested the choice of treatments to the patient and his family: partial resection to obtain pathological diagnosis, complete resec- tion to obtain total cure, sclerotherapy, laser therapy, and conservative therapy. Additionally, we communicated surgical complications, especially massive bleeding, nerve palsy, and postoperative airway obstruction. They decided on the course of complete resection.

\section{Operation Findings}

Although we had configured an S-shaped incision extending the line to midline jaw dissection as occasion arises, we could obtain a wide surgical view with a simple arcuate skin incision under the mandible followed by removal of the submandibular gland and cut off the digastric and stylohyoid muscle. At the back side of the submandibular bone, we found the caudal side of the tumor. As it had no adhesion, we peeled the tumor from the surroundings with blind-finger dissection and exposed internal and external carotid arteries in the deep portion. After recognition of arterial branches and hypoglossal nerve, we cut off the occipital and maxillary arteries excluded to the caudal side of the medial pterygoid muscle. As the tumor had shrunk after ligations of the maxillary artery, we could complete the resection without any complications ( - Fig. 3). After resection, we repaired the disconnected digastric muscle and recognized the internal and external carotid arteries, jugular vein, and hypoglossal nerve and then preserved. The sympathetic chain, vagus nerve, accessary nerve, and glossopharyngeal nerve did not appear in the surgical view. The operation time was 249 minutes, and intraoperative blood loss was approximately $100 \mathrm{~mL}$.

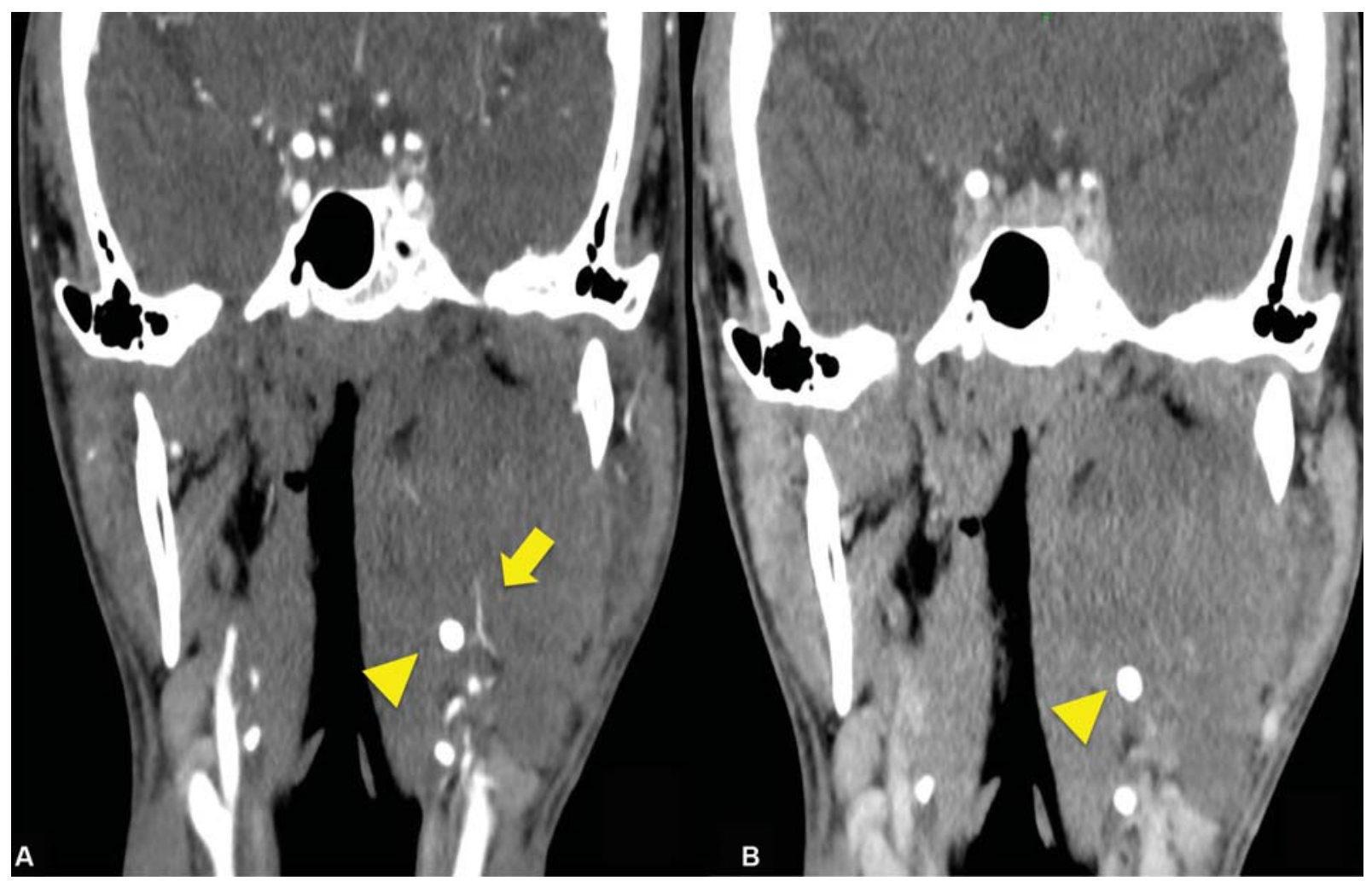

Fig. 1 Dynamic enhanced computed tomography. (A) In arterial enhanced phase, the tumor had no enhanced effect and calcification spot inside (arrowhead). A branch of facial artery was detected running through the tumor (arrow). (B) In venous enhanced phase, the tumor had some enhanced effects and calcification spot inside (arrowhead). 

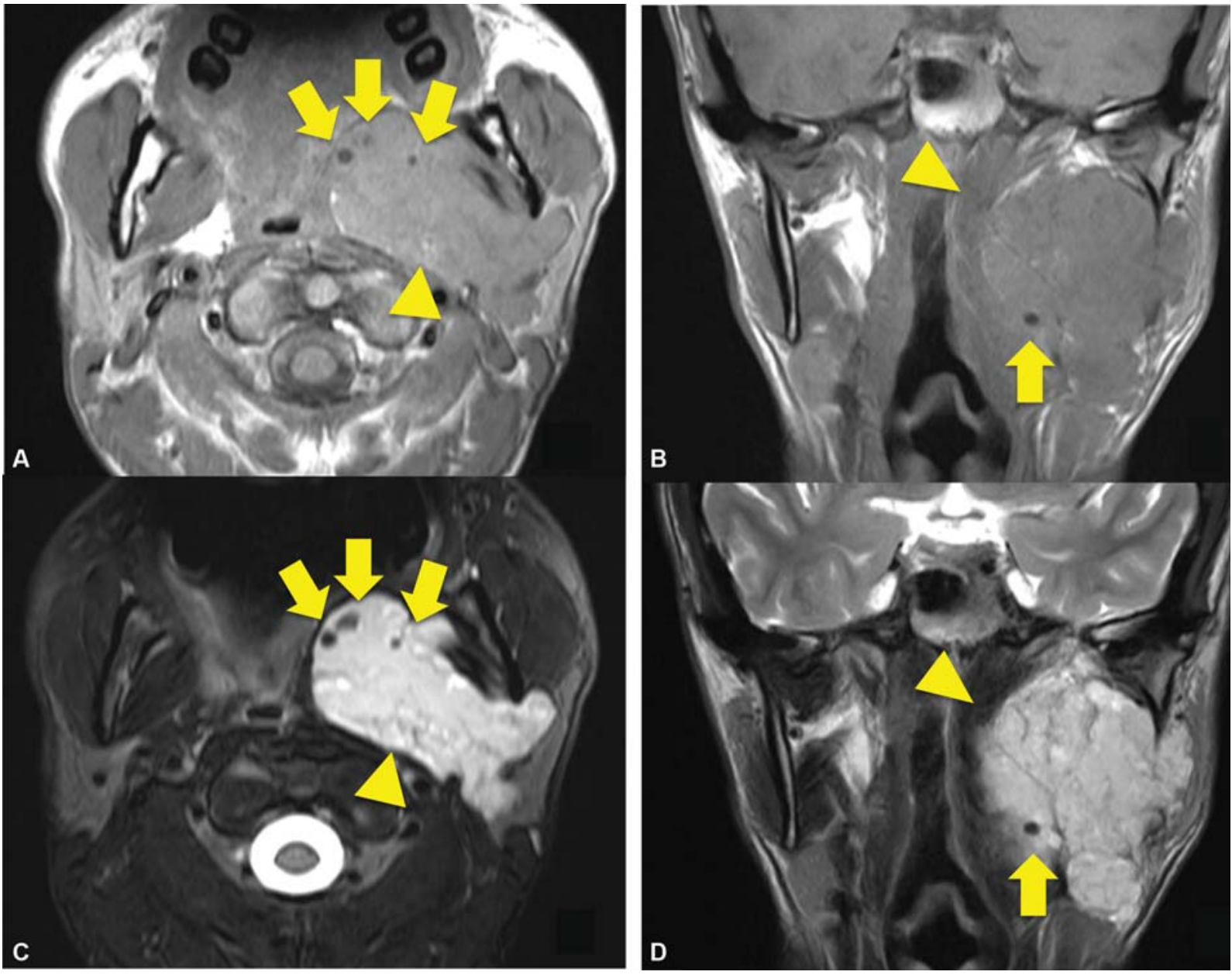

Fig. 2 Magnetic resonance imaging (MRI). The tumor was isointense overall on the axial (A) and coronal (B) T1-weighted images. The tumor signal ranged from iso- to hyperintense on the axial (C) and coronal (D) T2-weighted images. A clear margin from the surrounding tissues is seen (arrowheads), and calcifications were detected as areas of very low signal on both T1- and T2-weighted images (arrows).

\section{Pathological Findings}

Hematoxylin and eosin staining showed that the tumor contained hyperplasia of vessels, organizing blood clot, ossification, and hemosiderin deposit. Pathological diagnosis was venous malformation: cavernous angioma ( - Fig. 4).

After surgery, the patient was on a good clinical course without trismus and discharged from the hospital on postoperative day 8 . The patient showed no recurrence after 2 years of treatment.

\section{Discussion}

Venous malformations are the most common subtype of vascular malformation and represent 44 to $64 \%$ of vascular malformations. They can be localized or diffuse and can occur in more than one body site: extremity (40\%), head and neck (40\%), and trunk of the body (20\%). ${ }^{5}$ Faruque et al reported in a systematic review of 1,143 parapharyngeal space tumors that a majority (82\%) were benign and hemangioma was present in only 11 cases $(0.96 \%){ }^{6}$ In Japan, Okamoto et al reported on the pathological diagnosis of 76 parapharyngeal space tumors, and they found that majority were schwannomas $(42 \%)$ or pleomorphic adenomas (37\%) and there were no venous malfor- mations. ${ }^{7}$ There were few reports of venous malformations in the parapharyngeal space. Meanwhile, Maeda et al reported a case experience with information that they had difficulty in preoperative diagnosis from image findings. ${ }^{8}$

Venous malformations are characterized by a soft, compressible, no pulsatile tissue mass and have several forms: cystic, varix-like, dilated vessel-like, and sponge-like forms. ${ }^{9}$ Rapid enlargement may occur in certain situations such as surgery, trauma, infection, or hormonal changes associated with puberty, pregnancy, or menopause. Departed from vascular tumor, venous malformations can be confined to one specific area or spread out, and it can be superficial or deep. ${ }^{10}$ Deep cutaneous or intramuscular lesions usually cause discomfort, often in the early morning on awakening or with exertion. Intraoral venous malformations can bleed, distort dentition, cause speech problems, and obstruct the upper airway. Thrombosis, swelling, and pain are common in venous malformations. Only symptomatic malformations or lesions causing important aesthetic prejudice require treatment. ${ }^{11}$

History and physical examination remain the mainstay of diagnosis, which is made by clinical assessment. Imaging is reserved to evaluate the depth and extent of the lesion. Doppler ultrasound (US) is essential in differentiating venous 


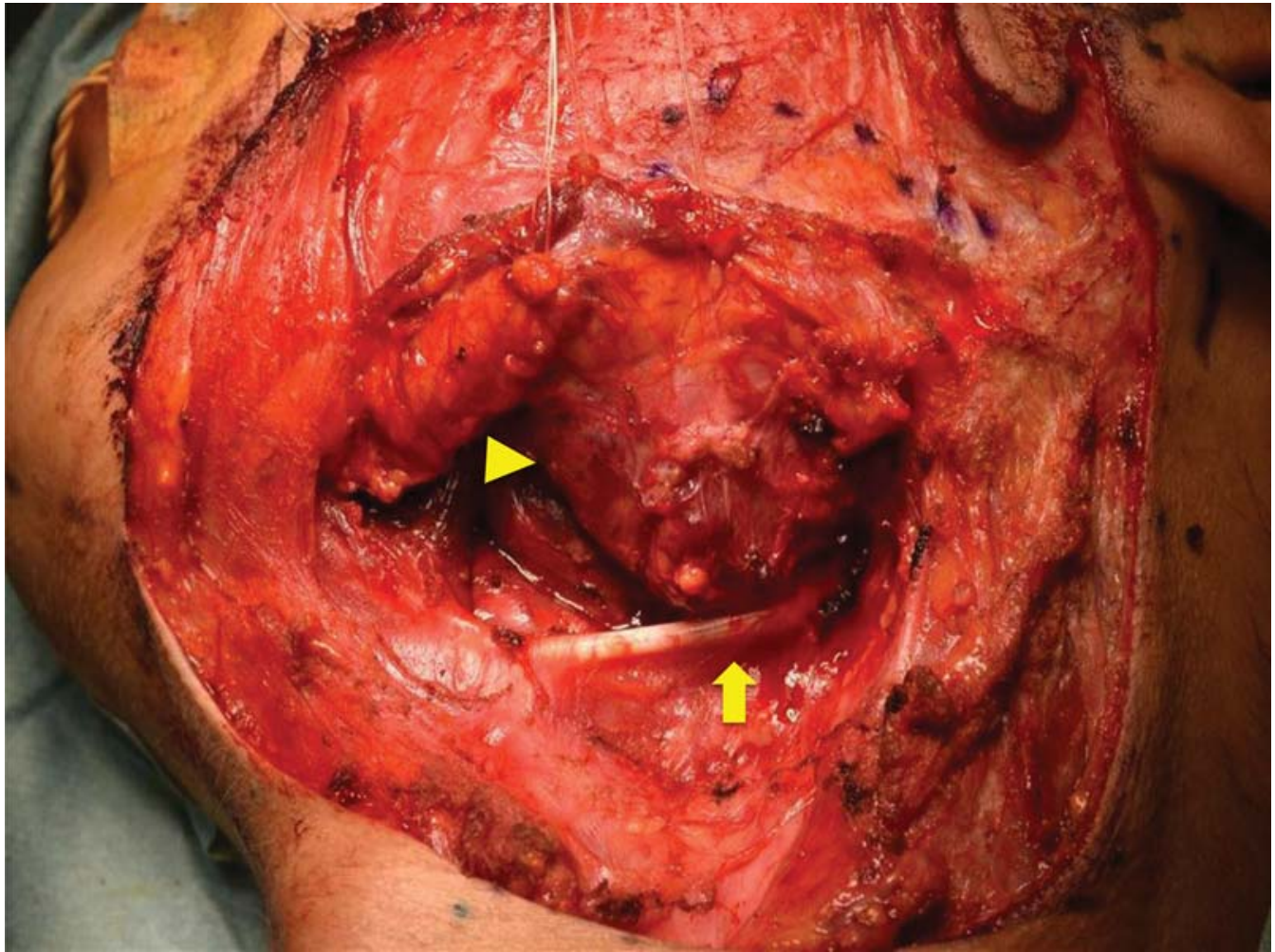

Fig. 3 Photograph of the operative field after removal of submandibular gland during tumor resection. Caudal side of the tumor (arrowhead) was located in the parapharyngeal space adjacent to the digastric tendon (arrow).

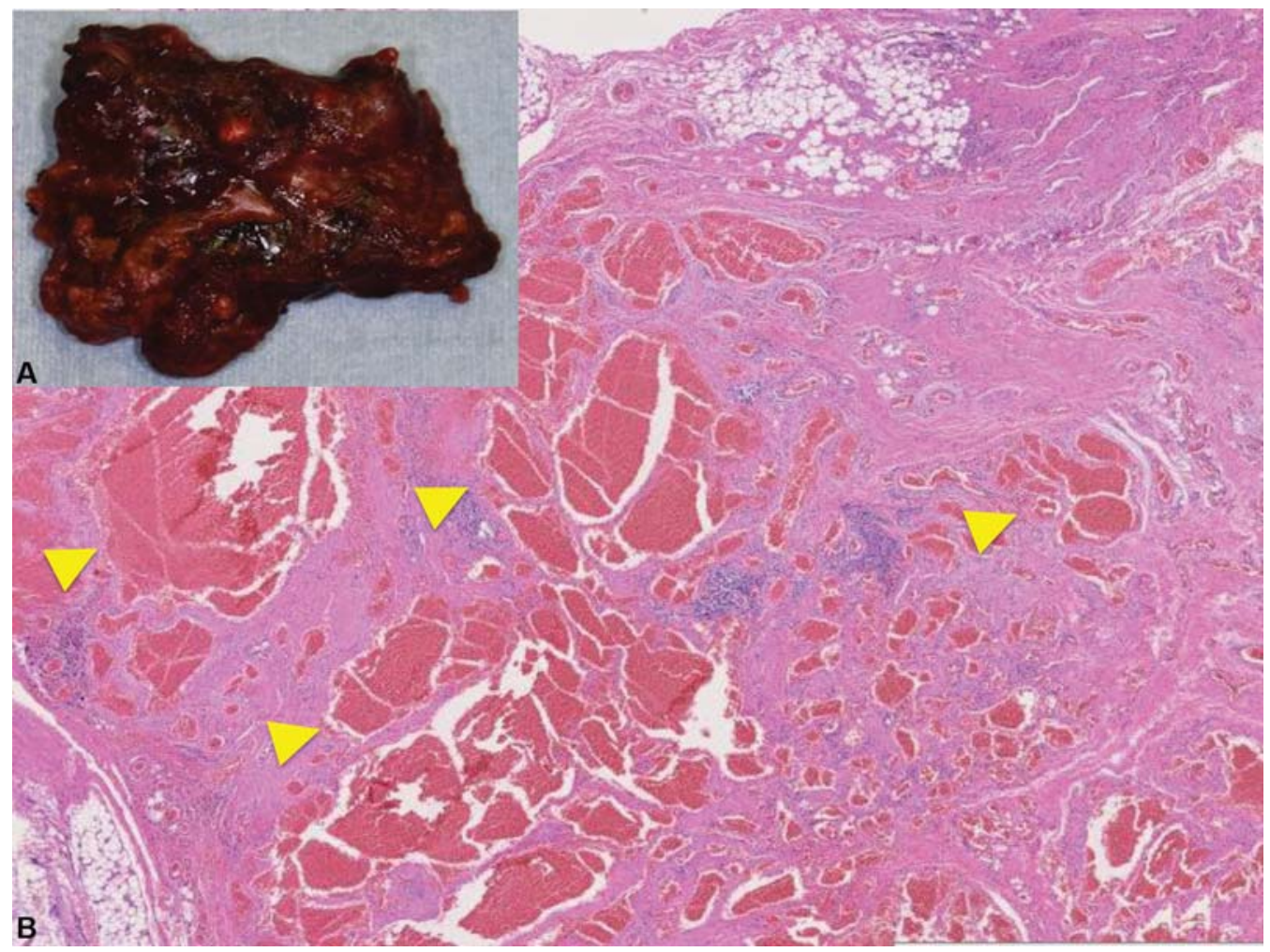

Fig. 4 Macroscopic and microscopic images of the tumor. (A) The excised specimen was dark brown in color with sponge-like appearance, and contracted in size after resection. (B) Hematoxylin and eosin staining of the tumor specimen showed hyperplasia of vessels, organized blood clots, calcifications, and hemosiderin deposits (arrowheads). 
malformations from other vascular anomalies. They appear as hypoechoic or heterogenous lesions in $80 \%$ of cases. ${ }^{12,13}$ Anechoic channels can be visualized in less than $50 \%$ of cases. Sometimes, isoechoic thickening of the subcutaneous tissues without a solid mass or discernible channel is the only feature. Hyperechoic foci with posterior acoustic shadowing are seen in less than $20 \%$ of cases. ${ }^{12}$ Although US is traditionally and sill currently used for characterization of vascular lesions, it is limited by a small field of view, restricted penetration, and operator dependency. At CT, venous malformations are hypoattenuating or heterogeneous lesions that enhance slowly and peripherally after bolus injection of contrast material. Phleboliths are more clearly depicted and fatty components are sometimes demonstrated at CT. ${ }^{14,15}$ MRI is excellent for defining the extension of vascular malformations and their relationship to adjacent structures. High-flow arteriovenous malformations and fistulas contain a signal void, whereas slow-flow venous malformations are usually hypo- or isointense at T1-weighted imaging and show high signal intensity on T2-weighted images. Areas of hypointensity related to thrombosis, septation inside, or phleboliths also can be observed. $^{16,17}$ Gadolinium-enhanced T1-weighted imaging is useful in evaluating the circulatory portion of the malformation. As venous malformations with a connection to deep veins can be a high risk factor of venous clot, delayed phaseenhanced MRI is essential for establishing a treatment strategy. $^{18}$

Venous malformations that are not causing symptoms do not require treatment. Basic treatment consists of the use of graded elastic compression stockings or sleeves to prevent swelling and formation of clot or phlebolith. ${ }^{12}$ However, especially in head and neck regions, compression can be adapted for few cases because of anatomical location. Conservative treatment options are sclerotherapy, laser therapy, venous embolization, and surgical excision. ${ }^{19,20}$

Although sclerotherapy is effective for small marginal lesions that have less complications than radical surgery for cosmetic aspect, deep and large lesions require performing the procedure several times to achieve complete and permanent shrinkage. ${ }^{19,20}$ For patients with extensive venous malformation in the parapharyngeal space, it is necessary to consider the possibility of airway compromised and systemic complications depending on the sclerosing agent after the procedure. Laser therapy is similar to sclerotherapy and it is useful for treating very superficial lesions, such as oral, tongue, and pharyngeal lesions. ${ }^{21}$

Surgical excision is generally contemplated after sclerotherapy when treatment is incomplete or when an aesthetic prejudice requires correction. A superior aspect of surgery is removing all of the abnormal veins and phleboliths. However, surgery also has complications of massive bleeding, nerve palsy, and cosmetic aspect. ${ }^{10,22}$ We have to select an adequate treatment strategy for head and neck lesions.

In this case, we suspected the diagnosis of venous malformation based on the examinations. As it was expected that multiple sclerotherapy would be needed to obtain complete shrinkage and could be a cause of airway edema, we eliminated sclerotherapy for initial treatment for this case. As a relatively bulky venous malformation was located under mucosa in the pharyngeal lesion, we considered laser therapy was inadequate. Fortunately, we could perform radical resection without complications. As three-dimensional angiographic CT scans were performed and there were some enhanced effects in the tumor, we did not select a preoperative angiography to avoid invasiveness of the exam. However, according to our operation findings, angiography would be useful for detecting feeding vessel and intravascular embolus even for venous malformation. The vascular malformation in the head and neck regions should be diagnosed before primary treatment with multimodality as they are linked to selection of treatment strategy. Additionally, we have to choose adequate treatment based on the location and conditions of vascular malformation with certain considerations.

\section{Conclusion}

We experienced a unique case of huge venous malformation in the head and neck regions. As venous malformation in the parapharyngeal space is uncommon and treatment strategies have not been established, further investigations are required to obtain a definitive treatment strategy.

Conflict of Interest

None declared.

\section{References}

1 Hassanein AH, Mulliken JB, Fishman SJ, Greene AK. Evaluation of terminology for vascular anomalies in current literature. Plast Reconstr Surg 2011;127(01):347-351

2 Mulliken JB, Glowacki J. Hemangiomas and vascular malformations in infants and children: a classification based on endothelial characteristics. Plast Reconstr Surg 1982;69(03):412-422

3 Mulliken JB, Young AE. Vascular Birthmarks: Hemangiomas and Malformations. Philadelphia, PA: WB Saunders; 1988

4 Enjolras O, Mulliken JB. Vascular cutaneous anomalies in children: malformations and hemangiomas. Pediatr Surg Int 1996;11 (5-6):290-295

5 Loose DA. Surgical management of venous malformations. Phlebology 2007;22(06):276-282

6 Riffat F, Dwivedi RC, Palme C, Fish B, Jani P. A systematic review of 1143 parapharyngeal space tumors reported over 20 years. Oral Oncol 2014;50(05):421-430

7 Okamoto I, Kamata S, Miura K, et al. A site of origin histopathological study on parapharyngeal space tumors. A review of 76 cases [in Japanese]. Nippon Jibiinkoka Gakkai Kaiho 2013;116 (01):27-30

8 Maeda H, Touyama M, Agena S, Matayoshi S. A surgical treatment of cavernous haemangioma in the parapharyngeal space. Head Neck Surg 2016;26(01):109-113

9 Mulliken JB, Fishman SJ, Burrows PE. Vascular anomalies. Curr Probl Surg 2000;37(08):517-584

10 Dompmartin A, Vikkula M, Boon LM. Venous malformation: update on aetiopathogenesis, diagnosis and management. Phlebology 2010;25(05):224-235

11 Dubois J, Soulez G, Oliva VL, Berthiaume MJ, Lapierre C, Therasse E. Soft-tissue venous malformations in adult patients: imaging and therapeutic issues. Radiographics 2001;21(06):1519-1531

12 Trop I, Dubois J, Guibaud L, et al. Soft-tissue venous malformations in pediatric and young adult patients: diagnosis with Doppler US. Radiology 1999;212(03):841-845 
13 Paltiel HJ, Burrows PE, Kozakewich HP, Zurakowski D, Mulliken JB. Soft-tissue vascular anomalies: utility of US for diagnosis. Radiology 2000;214(03):747-754

14 Kakimoto N, Tanimoto K, Nishiyama H, Murakami S, Furukawa S, Kreiborg S. CT and MR imaging features of oral and maxillofacial hemangioma and vascular malformation. Eur J Radiol 2005;55 (01):108-112

15 Dompmartin A, Vikkula M, Boon LM. Venous malformation: update on aetiopathogenesis, diagnosis and management. Phlebology 2010;25(05):224-235

16 Legiehn GM, Heran MK. Venous malformations: classification, development, diagnosis, and interventional radiologic management. Radiol Clin North Am 2008;46(03):545-597, vi

17 Moukaddam H, Pollak J, Haims AH. MRI characteristics and classification of peripheral vascular malformations and tumors. Skeletal Radiol 2009;38(06):535-547
18 Vilanova JC, Barceló J, Smirniotopoulos JG, et al. Hemangioma from head to toe: MR imaging with pathologic correlation. Radiographics 2004;24(02):367-385

19 Heit JJ, Do HM, Prestigiacomo CJ, et al; SNIS Standards and Guidelines committee. Guidelines and parameters: percutaneous sclerotherapy for the treatment of head and neck venous and lymphatic malformations. J Neurointerv Surg 2017;9(06): 611-617

20 Steiner F, FitzJohn T, Tan ST. Surgical treatment for venous malformation. J Plast Reconstr Aesthet Surg 2013;66(12): 1741-1749

21 Frigerio A, Tan OT. Laser applications for benign oral lesions. Lasers Surg Med 2015;47(08):643-650

22 Marler JJ, Mulliken JB. Current management of hemangiomas and vascular malformations. Clin Plast Surg 2005;32(01):99-116, ix 